\title{
Adding Relational Query Facilities to Software Development Environments
}

\author{
SUSAN HORWTTZ \\ University of Wisconsin-Madison
}

Software development environments should include query handlers. Query handlers based on the relational database model are attractive because the model provides a uniform, non-procedural approach to query writing. There are two drawbacks to using the relational model to support query handlers in software development systems: (1) Standard relational database systems require that all information be stored in relations; however, the data structures used by existing software development environments are generally non-relational, and it is impractical to replace them with relations. (2) The standard relational operators are not powerful enough to express certain important classes of queries.

In [10] we proposed a model of editing environments, based on the use of relationally-attributed grammars, that supports a relational query facility. We introduced a new kind of relations, implicit relations, and a new approach to query evaluation to handle queries that use implicit relations.

In this paper we illustrate the utility of implicit relations in contexts other than relationally-attributed grammars. We extend the definition of implicit relations and show how they can be used to support relational query facilities in software development environments without giving up the use of nonrelational data structures. Implicit relations can also be used to provide non-standard relational operations such as transitive closure.

\section{INTRODUCTION}

It is easy to see the benefits of including query facilities in components of software development environments, such as language-based editors, debuggers, and version-control managers. However, existing systems that provide query facilities generally do so in a limited way; the user is restricted to a pre-defined set of queries, and query answers cannot be used as inputs to further queries. This is because the query facilities are implemented in an ad hoc manner. A better approach would be to take advantage of current database technology, basing the query facility on the relational database model [4]. Under this model, arbitrary queries can be written by

This work was supported in part by the National Science Foundation under grant DCR-8603356.

Author's address: Computer Sciences Department, University of Wisconsin - Madison, 1210 W. Dayton St., Madison, WI 53706. 
applying a standard set of operators to a set of relations. Query answers are themselves relations, thus are available as inputs to further queries. An advantage of the relational model over the hierarchical and network database models is that the relational model provides a uniform, non-procedural approach to query writing.

Two systems that provide query facilities based on the relational database model are Omega [12] and Masterscope [15]. Omega is a language-based programming environment in which all program information is represented using relations. This representation has the advantage of allowing queries about program structure to be written; unfortunately, some traditional editing operations became unacceptably slow when performed using the relational representation. For example, using the standard INGRES relational database management system [19], the display of a ten-line procedure body required forty seconds of elapsed time. Even using a version of INGRES specially tuned for this task, the display required seven seconds. By contrast, display of such procedures by language-based editors that use more traditional tree data structures is virtually instantaneous.

Masterscope, which provides a query handler to users of the Interlisp system [20], takes a different approach. Some information is maintained explicitly in relations; other information is computed as needed, either from existing relations or from Interlisp data structures.

Our goal has been to generalize the approach taken in Masterscope so that a relational query facility can be added to any existing software-development environment, without requiring that fundamental data structures be replaced with relations. Our general model for relational query facilities relies on the use of implicit relations, introduced in [10] and extended herein. From the query-writer's point of view, an implicit relation is indistinguishable from any other relation; however, implicit relations are not stored as sets of tuples, instead the informational content of an implicit relation is computed as needed during query evaluation from stored relations, from non-relational data structures, or using pure mathematical functions.

The key to the use of implicit relations is the definition of a query-evaluation method that treats uniformly both implicit and "normal" (henceforth called explicit) relations [10]. This query-evaluation method uses three functions, a membership-test, a selective-retrieval, and a relation-producing function, to access all relations, both implicit and explicit, used in the query.

The remainder of the paper is organized as follows: Section 2 describes how membership-test, selective-retrieval, and relation-producing functions can be used to provide a query-evaluation method that handles queries with both implicit and explicit relations. Section 3 uses a hypothetical program-development environment that includes an interpreter to clarify the concept of implicit relations. The three approaches to defining implicit relations are illustrated; implicit relations are defined (1) using operations on a non-relational data structure, (2) using nonrelational operations on a stored relation, and (3) using pure mathematical functions. Queries that use these implicit relations are defined, and their evaluation using the method outlined in Section 2 is discussed. Section 4 compares the ideas presented in this paper with previous work. 


\section{QUERY-EVALUATION USING ACCESS FUNCTIONS}

The query-evaluation method presented in this section relies on queries being represented as expression trees (examples appear in Figures 1 and 2). Each node of a tree represents a relation: leaf nodes represent implicit or explicit relations, and internal nodes represent intermediate relations. The key to the evaluation method is the use of three access functions, membership test, selective retrieval, and relation producing functions:

\section{Definition:}

(1) The membership-test function for relation $\mathrm{R}$, given tuple $t$, returns true if $t$ is in $\mathrm{R}$, and otherwise returns false.

(2) The selective-retrieval function for relation $R$, given list of fields $f_{1}, f_{2}, \ldots f_{n}$, and list of values $v_{1}, v_{2}, \ldots v_{n}$, returns the set of tuples in $\mathrm{R}$ that have value $v_{i}$ in field $f_{i}$ for all $i$.

(3) The relation-producing function for relation $\mathrm{R}$ returns the set of tuples in relation $\mathrm{R}$.

Every node of the query tree has a membership-test, a selective-retrieval, and a relationproducing function associated with it. Access functions for implicit relations are provided as part of their definition; examples are given in Section 3. Access functions for explicit relations are also determined when the relations are defined, and depend on the specified storage and access methods. Access functions for internal nodes of the query tree are built as part of query evaluation; a function at node $n$ can call any of the functions associated with the children of $n$ in the tree.

A query is evaluated by calling the relation-producing function associated with the root node of the query tree. The success of the query-evaluation method depends on the use of membership-test and selective-retrieval functions to avoid materializing both implicit and intermediate relations. For example, the intersection and set difference operators can use an operand relation's membership-test function in place of an explicit representation of the operand relation. Given an explicit representation of relation R1, and a membership-test function for relation $R 2$, one can implement ( $R 1 \cap R 2)$ or ( $1-R 2)$ as follows: a single scan is made through $R 1$ considering each tuple $t$ in turn; $t$ is in (R1 $\cap \mathrm{R} 2)$ if it is a member of R2; $t$ is in (R1-R2) if it is not a member of $\mathbf{R} 2$.

Similarly, equi-join can be implemented using one explicit operand relation and the other operand relation's selective-retrieval function. A single scan is made through the explicit operand relation; for each tuple $t$, the selective-retrieval function of the other operand relation is called with the number of the join field and the appropriate value from $t$. All returned tuples are joined with $t$ and added to the result relation.

The use of a membership-test function to implement intersection or set difference, or of a selective-retrieval function to implement equi-join is essentially equivalent to the use of an index when the relation being accessed is a materialized relation. The important innovation introduced by our technique is that this relation need not be materialized; it can be an implicit relation, or can represent an arbitrary relational computation. Membership-test and selective- 
retrieval functions for intermediate relation $R$ can often be implemented so that neither $R$ itself nor any of the intermediate relations involved in its computation need to be built. Instead, the membership-test or selective-retrieval functions associated with the nodes of the subtree rooted at the node representing relation $\mathrm{R}$ are called. These calls propagate down the query tree until the membership-test or selective-retrieval functions provided for the implicit and/or explicit relations named at the leaves of the tree are called.

Figure 1 illustrates an example query with an intersection operator at its root. As discussed above, the relation-producing function associated with the intersection node can call either of its children's relation-producing functions and then, for each tuple $t$ in the result, call the other child's membership-test function. The select node's membership-test function can be implemented as follows: given tuple $t$, return true if and only if $t$ satisfies the selection predicate and $t$ is in relation R3 (determined by calling the membership-test function of the select node's child - the root of the subtree that defines R3). Similarly, the union node's membership-test function can be implemented by calling its children's membership-test functions; tuple $t$ is in the relation represented by the union node if it is in either $R 1$ or $R 2$.

Further examples of query trees and of their evaluation using membership-test and selectiveretrieval functions are given in Section 3. Details of the evaluation method can be found in [9] and [10].

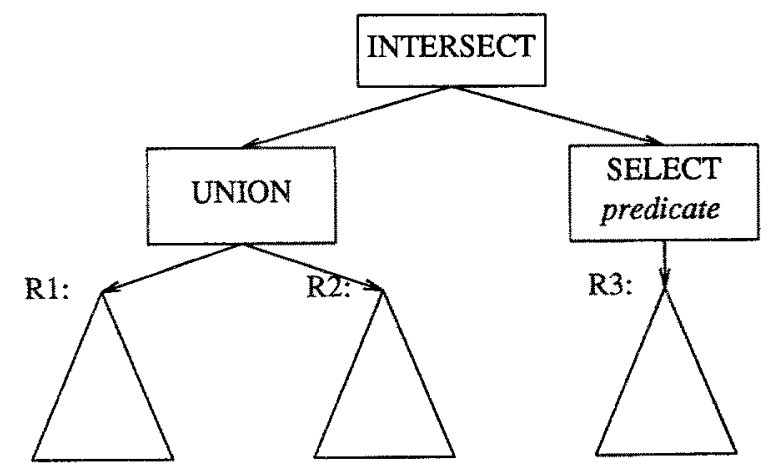

Figure 1. Example query tree. Relations R1, R2, and R3 are intermediate relations whose actual definitions are omitted. 


\section{IMPLICIT RELATIONS: EXAMPLES FROM PROGRAM-DEVELOPMENT ENVIRONMENTS}

In this section we illustrate the three ways that implicit relations can be defined: using arbitrary operations on non-relational data structures, using arbitrary operations on stored relations, and using pure mathematical functions. In each case we consider how the three access functions membership-test, selective-retrieval, and relation-producing functions - would be defined. We define queries that use our example implicit relations, and discuss how the queries would be evaluated using the technique outlined in Section 2. A program-development environment that includes an interpreter is used as the basis for our examples.

\subsection{Defining implicit relations using non-relational data structures}

Providing a query facility as part of an interpreter allows a programmer to ask questions about a program as it executes. An important class of questions are those that deal with the current state of the runtime stack. For example, assuming that access to non-local variables is handled using links maintained in the stack, a query that uses information from the stack is: "What are the current values of all global variables used by the currently active procedure?". Answering this question requires information about the current global environment as well as information about which variables are used but not declared in each procedure.

As discussed in Section 1, it is probably impractical to replace the traditional runtime stack representation with a relational representation ( $c f[5]$ ). While it may be reasonable to store in an explicit relation information about the set of global variables used by each procedure (since this information does not change as the program executes), it is probably not reasonable to maintain an explicit relational representation of the current global environment. Instead, the global environment can be defined as an implicit relation whose schema is:

\section{GlobalEnv (variable name, current value).}

The information that is conceptually stored in the GlobalEnv relation is actually stored in the runtime stack. Membership-test, selective-retrieval, and relation-producing functions are provided as part of the definition of the GlobalEnv relation. These access functions all involve traversals of the stack, following access links. The membership-test function, given a variable name and a value, follows links backward in the stack, searching for a local data field labeled with the given name. The function returns true if and only if such a field is found and the stored value matches the given value. Similarly, the selective-retrieval function, given a variable name, searches for a local data field labeled with that name, and returns the corresponding value. Given a value, the selective-retrieval function searches back through the entire stack, building up a relation containing the names of all visible variables that have the given value. The relation-producing function must also traverse the entire stack, building the complete GlobalEnv relation. 
The question "What are the current values of all global variables used by the currently active procedure?", can be formulated as a relational query using the implicit GlobalEnv relation and an explicit relation containing information about global variable usage:

\section{GlobalsUsed( procedure name, variable name).}

Figure 2 shows the example query in tree form.

Figure 3 shows an example program (consisting of three procedures: main, DeclaresXUsesY, and UsesXY), the corresponding GlobalsUsed relation, the runtime stack as it would appear after the call to UsesXY from DeclaresXUsesY, and the relation produced by evaluating the query of Figure 2 for the given program and runtime stack. For simplicity, we assume that the programming language in use uses dynamic scoping, and our illustration of the runtime stack includes only control-link and local data fields.

To evaluate this example query using the method outlined in Section 2, membership-test, selective-retrieval, and relation-producing functions are built for each internal node of the query

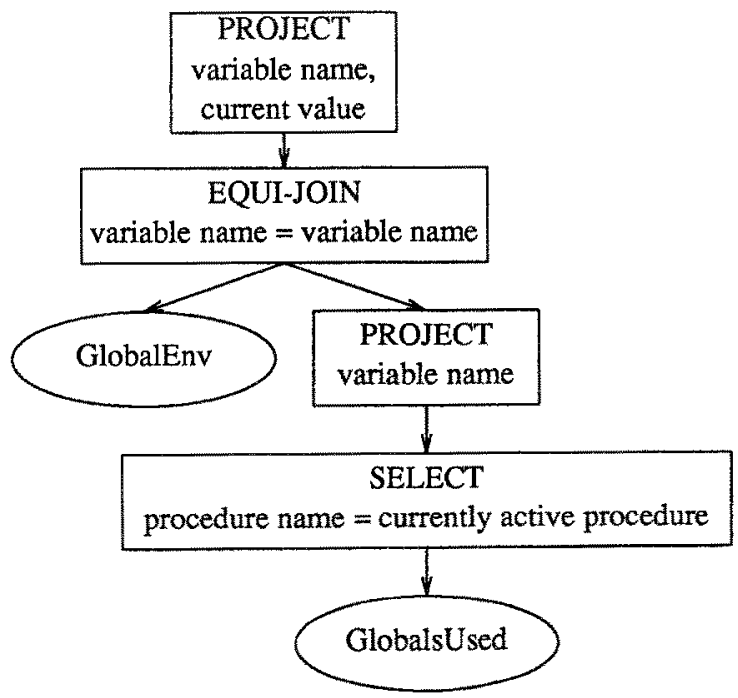

Figure 2. Example query tree: "What are the current values of the global variables of the currentlyactive procedure?". 


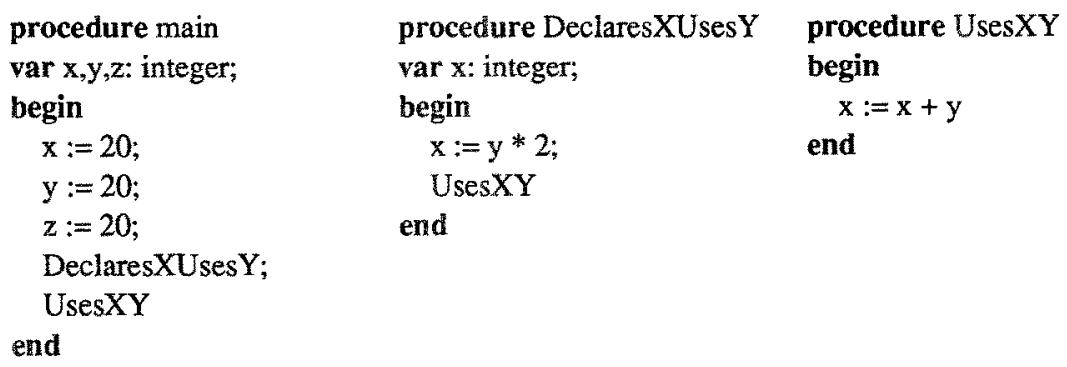

\begin{tabular}{|l|lc|} 
GlobalsUsed & procedure name & variable name \\
\hline & DeclaresXUsesY & $\mathrm{y}$ \\
UsesXY & $\mathrm{x}$ \\
UsesXY & $\mathrm{y}$ \\
\hline
\end{tabular}

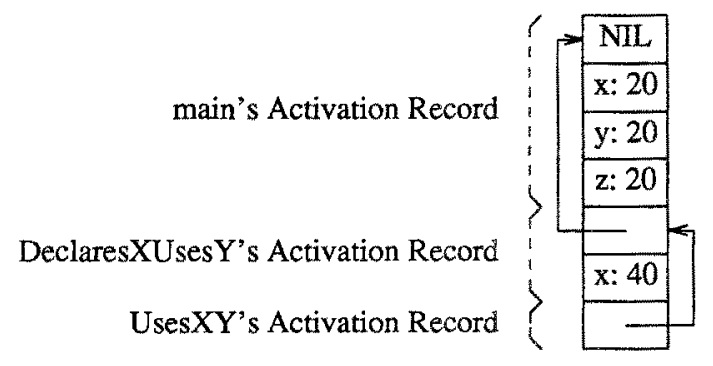

Figure 3. Example program, corresponding GlobalsUsed relation, the runtime stack after DeclaresXUsesY calls UsesXY, and the answer to the query "What are the current values of the global variables of the currently-active procedure?'. Each activation record on the stack includes a control link field and a field for each local variable. 
tree, and the relation-producing function of the root node is called. This function calls the relation-producing function associated with the equi-join node, which may perform any of the following actions:

(1) Call the relation-producing functions associated with each of the children of the equi-join node, and join the results.

(2) Call the relation-producing function associated with the GlobalEnv implicit relation; for each tuple $t$ in the result, call the selective-retrieval function associated with the project node, and join $t$ with the result of this call.

(3) Call the relation-producing function associated with the project node; for each tuple $t$ in the result, call the selective-retrieval function associated with the GlobalEnv implicit relation, and join $t$ with the result of this call.

For the trivial example illustrated in Figure 3, any of these three possibilities will do. However, the cost of calling GlobalEnv's relation-producing function is proportional to the depth of the runtime stack; thus, the third approach, which uses GlobalEnv's selective-retrieval function in place of its relation-producing function, will be advantageous given a deep stack.

\subsection{Defining implicit relations using non-relational operations on explicit relations}

One disadvantage of the relational model is the limited power of the standard relational operators; certain computations of interest cannot be formulated as relational queries [3]. In this section we illustrate how implicit relations defined using non-relational operations on explicit relations can help to overcome this problem.

The query we wish to express is: "Which procedures, called transitively from the current procedure, set the value of variable $x$ ?".

As shown in Figure 4, this query can be expressed using two relations, one containing information about transitive calls:

\section{CallsTransitively (calling procedure, called procedure)}

and one containing information about which variables are set by each procedure:

AssignsTo(procedure name, variable name).

While it would be possible to maintain a CallsTransitively relation, it may be preferable to maintain only the unclosed Calls relation, and to define Calls Transitively as an implicit relation. Calls Transitively can be defined in terms of Calls using the following rules:

(1) CallsTransitively $(p, q)$ if Calls $(p, q)$.

(2) CallsTransitively $(p, q)$ if Calls $(p, r)$ and CallsTransitively $(r, q)$.

The definition of an implicit relation must include definitions of the relation's three access functions. The access functions for Calls Transitively are most easily defined as logic programs using the two rules given above. 


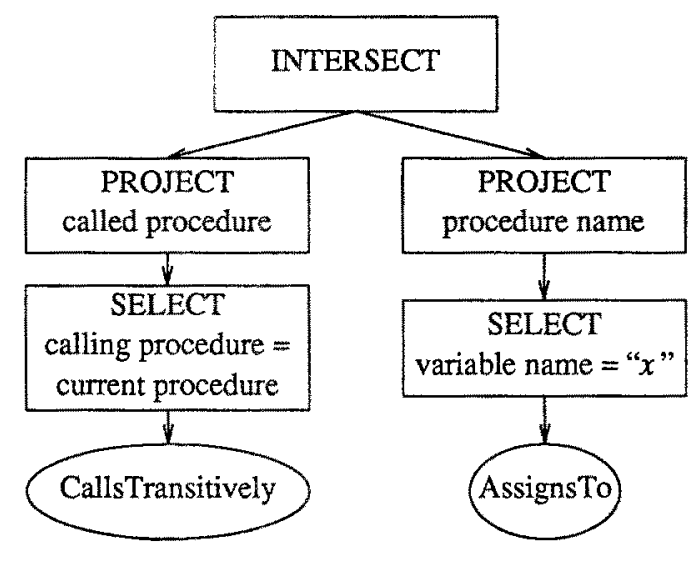

Figure 4. Example query tree: "Which procedures, called transitively from the current procedure, set the value of variable $x$ ?".

The AssignsTo relation could be an explicit relation; alternatively, if the programdevelopment environment maintains a tree representation of programs, the AssignsTo relation could be an implicit relation defined in terms of operations on the program tree.

The query shown in Figure 4 could be evaluated in a number of ways including the following:

(1) For every tuple $t$ in the intermediate relation represented by the right subtree of the query, the membership-test function of the left subtree of the query is called. This leads to a sequence of calls to CallsTransitively's membership-test function.

(2) Alternatively, CallsTransitively's selective-retrieval function could be called to compute the set of procedures called transitively from the current procedure; these procedure names would then be passed to the membership-test function of the right subtree of the query, leading to a sequence of calls to AssignsTo's membership-test function.

When Assigns To is an explicit relation, the choice of evaluation method depends on its size and access methods. When AssignsTo is an implicit relation defined in terms of the program tree, the first alternative requires a traversal of the entire tree; thus, the second alternative is probably preferable.

\subsection{Defining implicit relations using mathematical functions}

An example of an implicit relation with associated access functions defined using arithmetic operations is the (infinite) Plus relation: Plus(value1, value2, sum). Membership testing for 
Plus is trivial, as is selective retrieval as long as at least two fields are specified. How to handle selective retrieval when given only one field, and how to implement a relation-producing function is not obvious, and would depend on the context in which Plus is used. One possibility is to consider erroneous a query whose evaluation requires a call to Plus's relation-producing function or to Plus's selective-retrieval function with just one specified field. Another possibility is to produce one tuple at a time until some cut-off signal is given.

Although arithmetic operations like addition are supplied as aggregate functions in "real" database systems, they are not expressible using the standard relational operators. Defining Plus as an implicit relation provides a way to extend the power of the standard relational operators without requiring the use of aggregates. For example, given relation $\mathrm{R}$ with two integer fields: $R$ (intvalue1, intvalue2), it is possible to use the Plus relation to write a query that sums the fields of each tuple in $R$. This query is shown in Figure 5.

The join operation in this query is performed by calling Plus's selective-retrieval function once for every tuple in relation $R$. Because two fields are passed to the selective-retrieval function, the result is a single value, computed by adding the two given field values. Thus, the overall time required to evaluate the query is proportional to the size of $R$.

\section{RELATION TO PREVIOUS WORK}

In this paper, we have extended the definition of implicit relations introduced in [10]. In so doing, we provide the foundation for adding relational query facilities to software-development environments, in which it is impractical to store all information in relations. Related work falls

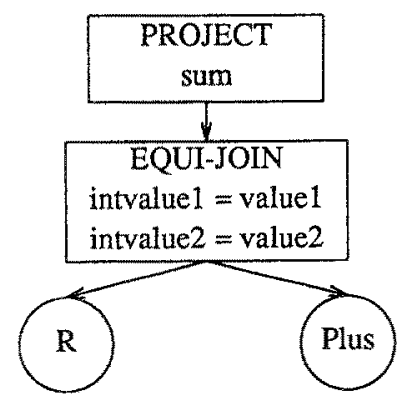

Figure 5. Example query: Sum the two fields of each tuple of relation $\mathbf{R}$. 
in three categories: (1) Other approaches to providing query facilities in development environments, (2) Concepts similar to implicit relations, and (3) Techniques similar to the queryevaluation method described here.

\subsection{Software-development systems with query facilities}

The most relevant previous work in this area [12], and [15] was discussed in Section 1. [11] describes another approach to providing a query facility during program development. Komorowski's approach is similar to Linton's, except that he stores program information in a Prolog database rather than in a relational database. Because Prolog's operators are inherently more powerful than the relational operators (for example, transitive closure can be expressed in Prolog but not using the standard relational operators), Komorowski's system can handle some queries that Linton's system cannot. The major weakness of Komorowski's system is that the database is not maintained incrementally; instead, programs are translated from a textual representation to Prolog form to allow interrogation of the database. An interesting area for future work would be to investigate the practicality of performing editing operations directly on the Prolog representation of the program.

\subsection{Implicit relations}

The concept of implicit relations has some similarities with the concept of views [21]. In both cases, the user of a database is given access to relations that are not actually stored as sets of tuples. View relations and implicit relations differ in how they can be defined, and in their intended use.

A view relation is defined by a query; conceptually, the query is re-evaluated after every modification to the database, and the view relation is the result of the most recent evaluation. View definitions are limited to relational operators applied to explicit or view relations.

By contrast, implicit relations are defined by three access functions: membership-test, selective-retrieval, and relation-producing functions. These access functions can be defined by applying arbitrary operators to arbitrary data structures; thus, implicit relations can be viewed as a generalization of view relations.

Implicit relations defined using non-relational operations on explicit relations have strong ties to logic programming. As noted in Section 3.2, logic programs may be the best way to define access functions for this class of implicit relations. While implicit relations provide a uniform approach to query writing and evaluation, the three access functions provided as part of the definition of an implicit relation can, in general, be implemented in an ad hoc fashion. The use of logic programming to implement these access functions provides a uniform approach at this level as well.

Logic programming does not, however, address the question of extracting information from non-relational data structures. Thus, implicit relations can be viewed as a more general concept, 
with logic programming providing a very important foundation for the definition of one class of implicit relations.

\subsection{Query evaluation using access functions}

The goals of our query-evaluation method are to avoid materializing both implicit and intermediate relations whenever possible. Having just considered the similarities between view relations and implicit relations, one is led to ask whether there is an analog to our first goal, avoiding the materialization of implicit relations, in the context of queries that use view relations. The answer is that query-evaluators do try to avoid the materialization of view relations used in queries. Because view relations are defined as relational operations on explicit relations, the mechanism for avoiding the materialization of view relations is straightforward: the view definition is inserted into the query in place of the view relation, and the entire query is optimized [18].

Of course, this approach will not work when an implicit relation is used in a query because implicit relations may not be defined in terms of relational operations on explicit relations. Thus, while the goal of avoiding the materialization of implicit relations is similar to the goal of avoiding the materialization of view relations, the methods used to achieve that goal must differ. Our approach to avoiding the materialization of implicit relations is to use the membership-test and selective-retrieval functions provided for these relations in place of the relations themselves.

Membership-test and selective-retrieval functions are also used to achieve our second goal, avoiding the materialization of intermediate relations. This can be viewed as a combination and generalization of the methods of [22] and [13].

Our use of selective-retrieval functions to evaluate joins corresponds to "tuple substitution" as presented in [22]. Our method can be considered to be a generalization of theirs because we are able to do lookups on non-materialized operand relations and because we allow the operands of joins to be defined using set operators, while they only consider queries defined using selection, projection, and join.

[13] proposes the use of membership tests for the evaluation of some set operators. However, because Liu was concerned with optimizing the execution of set-oriented programming languages, he considers only the set operators, and not selection, projection, or join. While it is rather trivial to build membership-test functions for all nodes of a query tree that contains only set operators, it is not obvious how to do so for a query tree that includes selection, projection, and join. Some of the subtleties that arise in the latter case are mentioned in [10]; a complete treatment appears in [9].

Other approaches to query optimization that seek to avoid building intermediate relations are the use of pipelining $[14,17,23]$ and of tree transformations $[8,17]$. When pipelining is used, a tuple in the result of one operation can be used as input to another operation as soon as it is produced, rather than waiting until an entire intermediate relation is formed. Pipelining is thus 
similar to our query-evaluation method in that intermediate relations are not materialized. It is dissimilar in that, when pipelining is used, the tuples of intermediate relations are all computed, whereas our method seeks to avoid or reduce the computation (as well as the materialization) of intermediate values. To understand this difference, consider the following query:

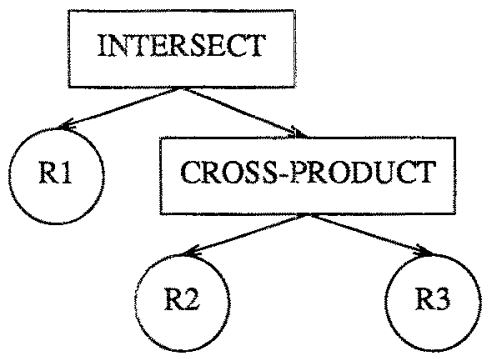

One way to evaluate this query without materializing the temporary relation represented by the CROSS-PRODUCT node is to use an index on relation R1 and to pipeline the result of the cross product. The cross product is computed a tuple-at-a-time, and for each tuple a lookup is done in R1's index; the tuple is in the result relation if it is in R1. While no temporary relation is materialized using this approach, all tuples of the cross product are computed.

By contrast, given indexes on relations R2 and R3, the query can be evaluated using our method without materializing the temporary relation, and without computing any of the tuples of the cross product: Relation R1 is scanned, each tuple $t$ is divided into two tuples, $t_{1}$ and $t_{2}$, according to the arities of R2 and R3, and lookups are done on $t_{1}$ and $t_{2}$ in the index for R2 and $\mathrm{R} 3$ respectively. Tuple $t$ is in the result relation if $t_{1}$ is in R2 and $t_{2}$ is in R3.

Of course, factors like the sizes of the three relations will determine which of the two methods is actually best; this example is merely meant to illustrate the philosophical difference between our approach and pipelining, and to indicate the potential advantages of our method. Further, the two approaches are not incompatible; pipelining can be incorporated into our approach by having selective-retrieval and relation-producing functions use pipelining rather than returning entire relations.

Tree transformations such as combining sequences of projections into a single projection and combining sequences of selections into a single selection, can reduce the number of intermediate relations represented by the internal nodes of the query tree; moving selection operators ahead of construction operators can reduce the sizes of these intermediate relations. These techniques do not, however, address the question of whether one can avoid building some of the intermediate relations of the transformed tree.

As with pipelining, incorporating tree transformations into our method will be important to its success. A query can often be represented using a number of different trees, and a different relation-producing function will be built for the root node of each such tree. It is important that 
transformations be used to produce trees in which the cost of the root node's relation-producing function is minimized.

Similarly, other optimization techniques could be used to produce more efficient membership-test, selective-retrieval, and relation-producing functions. Exploiting idempotency and unsatisfiability to simplify conditions $[6,8]$ can lead to more efficient membership-test and relation-producing functions; using the methods of $[1,2,16,23]$ for access path selection, the methods of [17] for choosing sort orders, and the methods of [7,22] for calculating join orders can all lead to more efficient relation-producing functions.

\section{SUMMARY}

Software development environments can include relational query facilities without giving up traditional, non-relational data structures, through the use of implicit relations. Information contained in non-relational data structures is conceptually stored as sets of tuples, and can be accessed by using implicit relations in queries. Implicit relations can also be defined using nonrelational operations on stored relations and using pure mathematical functions, thus indirectly extending the class of queries that can be expressed using the standard relational operators.

\section{REFERENCES}

1.

Astrahan, M. M. and D. D. Chamberlin, "Implementation of a structured English query language," Communications of the ACM 18(10) pp. 580-588 (Oct. 1975).

2.

Blasgen, M. W. and K. P. Eswaran, "Storage and access in relational databases," IBM Systems Journal 16(4) pp. 363-377 (1977).

3.

Chandra, A., "Programming primitives for database languages." Conference Record of the 8th ACM Symposium on Principles of Programming Languages, pp. 50-62 (Jan. 1981).

4.

Codd, E. F., "A relational model of data for large shared data banks," Communications of the ACM 13(6) pp. 377-387 (June 1970).

5.

Deutsch, L. P. and A. M. Schiffman, "Efficient implementation of the Smalltalk-80 system," Conference Record of the 11th ACM Symposium on Principles of Programming Languages, pp. 297-302 (Jan. 1984).

6.

Eswaran, K. P., J. N. Gray, R. A. Lorie, and I. L. Traiger, "The notions of consistency and predicate locks in a database system," Communications of the ACM 19(11) pp. 624-633 (1976).

7 .

Gotlieb, L. R., "Computing joins of relations," ACM SIGMOD International Conference on Management of Data, pp. 55-63 (May 1975).

8.

Hall, P. A. V., "Optimization of single expressions in a relational data base system," IBM J. Res. Develop. 20 pp. 244-237 (May 1976). 
9.

Horwitz, S., "Generating language-based editors: A relationally-attributed approach," TR 85-696 (Ph.D. thesis), Department of Computer Science, Comell University (Aug. 1985). 10.

Horwitz, S. and T. Teitelbaum, "Generating editing environments based on relations and attributes," ACM Transactions on Programming Languages and Systems 8(4) pp. 577-608 (Oct. 1986).

11.

Komorowski, H. J., "Rapid software development in a database framework - A case study," IEEE 1984 Proceedings of the International Conference on Data Engineering, (1984).

12.

Linton, M.A., "Implementing relational views of programs," Proceedings of the ACM SIGSOFT/SIGPLAN Software Engineering Symposium on Practical Software Development Environments, pp. 132-140 (Apr. 23-25, 1984).

13.

Liu, L., "Essential uses of expressions in set-oriented expressions," Ph.D. Thesis, Department of Computer Science, Cornell University (May 1979).

14.

$\mathrm{Lu}, \mathrm{H}$. and M. J. Carey, "Some experimental results on distributed join algorithms in a local network," Proceedings of VLDB, pp. 292-304 (1985).

15.

Masinter, L., "Global program analysis in an interactive environment," Ph.D. Thesis, Stanford University, Stanford, CA (Jan., 1980).

16.

Selinger, P. G., M. M. Astrahan, D. D. Chamberlin, R. A. Lorie, and T. G. Price, "Access path selection in a relational database management system," ACM SIGMOD International Conference on Management of Data, pp. 23-34 (May-June, 1979).

17.

Smith, J. M. and P. Chang, "Optimizing the performance of a relational algebra database interface," Communications of the ACM 18(10) pp. 568-579 (Oct. 1975).

18.

Stonebraker, M., "Implementation of integrity constraints and views by query modification," $A C M$ SIGMOD International Conference on Management of Data, pp. 65-78 (May 1975).

19.

Stonebraker, M., E. Wong, P. Kreps, and G. Held, "The design and implementation of INGRES," ACM Transactions on Database Systems 1(3) pp. 189-222 (Sept. 1976).

20.

Teitelman, W., Interlisp Reference Manual, Xerox PARC (Dec. 1978). (as cited in [Masinter 80]) 21.

Ullman, J., Principles of Database Systems, Computer Science Press, Potomac, MD (1980). 22.

Wong, E. and K. Youssefi, "Decomposition - a strategy for query processing," ACM Transactions on Database Systems 1(3) pp. 223-241 (Sept. 1976).

23.

Yao, S. B., "Optimization of query evaluation algorithms," ACM Transactions on Database Systems 4(2) pp. 133-155 (1979). 